\title{
Changes in the relationship between species richness and belowground biomass among grassland types and along environmental gradients in Xinjiang, Northwest China
}

\author{
YANG Yuling ${ }^{1,2}$, LI Minfei ${ }^{1}$, MA Jingjing ${ }^{1}$, CHENG Junhui ${ }^{1}$, LIU Yunhua ${ }^{1}$, JIA Hongtao ${ }^{1}$, LI \\ Ning $^{1}$, WU Hongqi ${ }^{1}$, SUN Zongjiu ${ }^{1}$, FAN Yanmin ${ }^{1}$, SHENG Jiandong ${ }^{1}$, JIANG Ping'an ${ }^{1 *}$ \\ ${ }^{1}$ Xinjiang Key Laboratory of Soil and Plant Ecological Processes, College of Grassland and Environmental Sciences, Xinjiang \\ Agricultural University, Urumqi 830052, China; \\ ${ }^{2}$ Xinjiang Institute of Ecology and Geography, Chinese Academy of Sciences, Urumqi 830011, China
}

\begin{abstract}
The association between biodiversity and belowground biomass (BGB) remains a central debate in ecology. In this study, we compared the variations in species richness (SR) and BGB as well as their interaction in the top $(0-20 \mathrm{~cm})$, middle $(20-50 \mathrm{~cm})$ and deep $(50-100 \mathrm{~cm})$ soil depths among 8 grassland types (lowland meadow, temperate desert, temperate desert steppe, temperate steppe desert, temperate steppe, temperate meadow steppe, mountain meadow and alpine steppe) and along environmental gradients (elevation, energy condition (annual mean temperature (AMT) and potential evapotranspiration (PET)), and mean annual precipitation (MAP)) based on a 2011-2013 survey of 379 sites in Xinjiang, Northwest China. The SR and BGB varied among the grassland types. The alpine steppe had a medium level of SR but the highest BGB in the top soil depth, whereas the lowland meadow had the lowest SR but the highest BGB in the middle and deep soil depths. The SR and BGB in the different soil depths were tightly associated with elevation, MAP and energy condition; however, the particular forms of trends in SR and BGB depended on environmental factors and soil depths. The relationship between SR and BGB was unimodal in the top soil depth, but SR was positively related with BGB in the middle soil depth. Although elevation, MAP, energy condition and SR had significant effects on BGB, the variations in BGB in the top soil depth were mostly determined by elevation, and those in the middle and deep soil depths were mainly affected by energy condition. These findings highlight the importance of environmental factors in the regulations of SR and $\mathrm{BGB}$ as well as their interaction in the grasslands in Xinjiang.
\end{abstract}

Keywords: species richness; belowground biomass; unimodal pattern; energy condition; soil depths; mean annual precipitation; grasslands in Xinjiang

Citation: YANG Yuling, LI Minfei, MA Jingjing, CHENG Junhui, LIU Yunhua, JIA Hongtao, LI Ning, WU Hongqi, SUN Zongjiu, FAN Yanmin, SHENG Jiandong, JIANG Ping'an. 2019. Changes in the relationship between species richness and belowground biomass among grassland types and along environmental gradients in Xinjiang, Northwest China. Journal of Arid Land, 11(6): 855-865. https://doi.org/10.1007/s40333-019-0068-8

\section{Introduction}

The relationship between biodiversity and productivity has been subject to major debate in

*Corresponding author: JIANG Ping'an (E-mail: jpa@xjau.edu.cn)

Received 2019-03-02; revised 2019-07-24; accepted 2019-08-23

(C) Xinjiang Institute of Ecology and Geography, Chinese Academy of Sciences, Science Press and Springer-Verlag GmbH Germany, part of Springer Nature 2019 
community ecology for a long time (Tilman, 1999; Hooper et al., 2005). It has been examined recently in many terrestrial ecosystems, including forests, grasslands and shrublands (Fraser et al., 2015; Chen et al., 2018; Ammer, 2019). However, most of these studies focus on the relationship between species richness (SR) and aboveground biomass (AGB), whereas studies on the relationship between SR and belowground biomass (BGB) remain rare (Allan et al., 2013). According to theoretical and empirical research, high SR can increase BGB via niche differentiation in rooting depth and differences in biomass allocation (De-Boeck et al., 2008; Turnbull et al., 2016). However, understanding on the relationship between SR and BGB remains controversial.

Three types of relationships between SR and BGB are recognized. In some broad-scale field studies and long-term controlled experiments, SR is linearly and positively related with BGB in a diversity of ecosystems, including forests, grasslands and shrublands (Mueller et al., 2013; Chen et al., 2018), indicating that communities with the higher SR also had a greater BGB because of niche differentiation. In some ecosystems, SR is not significantly associated with BGB, because in communities with a high SR, the overriding effect is on AGB while BGB remains constant (Bessler et al., 2009). Additionally, a unimodal pattern of relationship (increased firstly and then decreased) between SR and BGB is also observed in some studies (e.g., Zhu et al., 2017), suggesting that peak BGB appears at a medium level of SR, while the low BGB occurs under both low and high SR. The unimodal pattern is traditionally attributed to changes in ecological processes along an environmental gradient (Guo and Berry, 1998). For example, environmental filter only allows a few tolerant species to establish in resource-poor conditions, which in turn leads to lower SR and BGB (Guo and Berry, 1998). In resource-rich conditions, species with less competitive ability are gradually eliminated due to competition of species with high growth rates, which leads to the low SR but high BGB in the community (Guo and Berry, 1998). The conflicting findings may stem partly from regulations of SR and BGB by environmental factors (Loreau, 2000; Isbell et al., 2017). For example, both SR and BGB in forests, grasslands and shrublands of China are regulated by a climate gradient, but the direction and magnitude of the effects of the climate gradient on SR and BGB varied among ecosystem types (Cleland et al., 2013; Chen et al., 2018). Moreover, the heterogeneity of habitats in which communities are located also determines the relationship between SR and BGB. As habitat diversity changes from relatively homogeneous to heterogeneous, the relationship between SR and BGB shifts from positive, negative, or non-existent to unimodal (Guo and Berry, 1998). Thus, exploration on a broad scale with a diversity of habitats will greatly enhance understanding of the SR-BGB relationship.

In this study, the SR-BGB relationship was explored in the grasslands of Xinjiang Uygur Autonomous Region in Northwest China. The area of grasslands in Xinjiang is the third largest in China and the grasslands include a diversity of types (Xu, 1993), providing an ideal system to examine effects of habitat heterogeneity on SR-BGB relationship. Moreover, vertical zonality characterizes the grasslands in Xinjiang and the large variation in elevation produces a broad climate gradient, which is especially suitable for exploring the effects of environmental gradients on SR-BGB relationship. In particular, the following three questions were addressed: (1) how do SR and BGB vary among grassland types and along environmental gradients? (2) how does SR respond to the variation in BGB among grassland types? and (3) how do the environmental factors (elevation and climatic variables) affect the relationship of SR and BGB in the grasslands of Xinjiang?

\section{Materials and methods}

\subsection{Study area}

This research was performed in the grasslands of Xinjiang in Northwest China. The study area ranges from $34^{\circ} 25^{\prime} \mathrm{N}$ to $48^{\circ} 10^{\prime} \mathrm{N}$ in latitude and from $73^{\circ} 40^{\prime} \mathrm{E}$ to $96^{\circ} 18^{\prime} \mathrm{E}$ in longitude (Fig. 1), covering an area of $16.6 \times 10^{6} \mathrm{~km}^{2}$. The elevation ranges from 220 to $4700 \mathrm{~m}$ a.s.l. With increasing elevation, the mean annual precipitation (MAP) shifts from 10 to $550 \mathrm{~mm}$, and approximately $34 \%$ of MAP falls in the growing season. The annual mean temperature (AMT) changes from $9^{\circ} \mathrm{C}$ to $12^{\circ} \mathrm{C}$ with the decrease in elevation, and the lowest monthly mean temperature occurs in January $\left(-20^{\circ} \mathrm{C}\right)$ and the 
highest in July $\left(33^{\circ} \mathrm{C}\right)$ (Liu et al., 2016a).

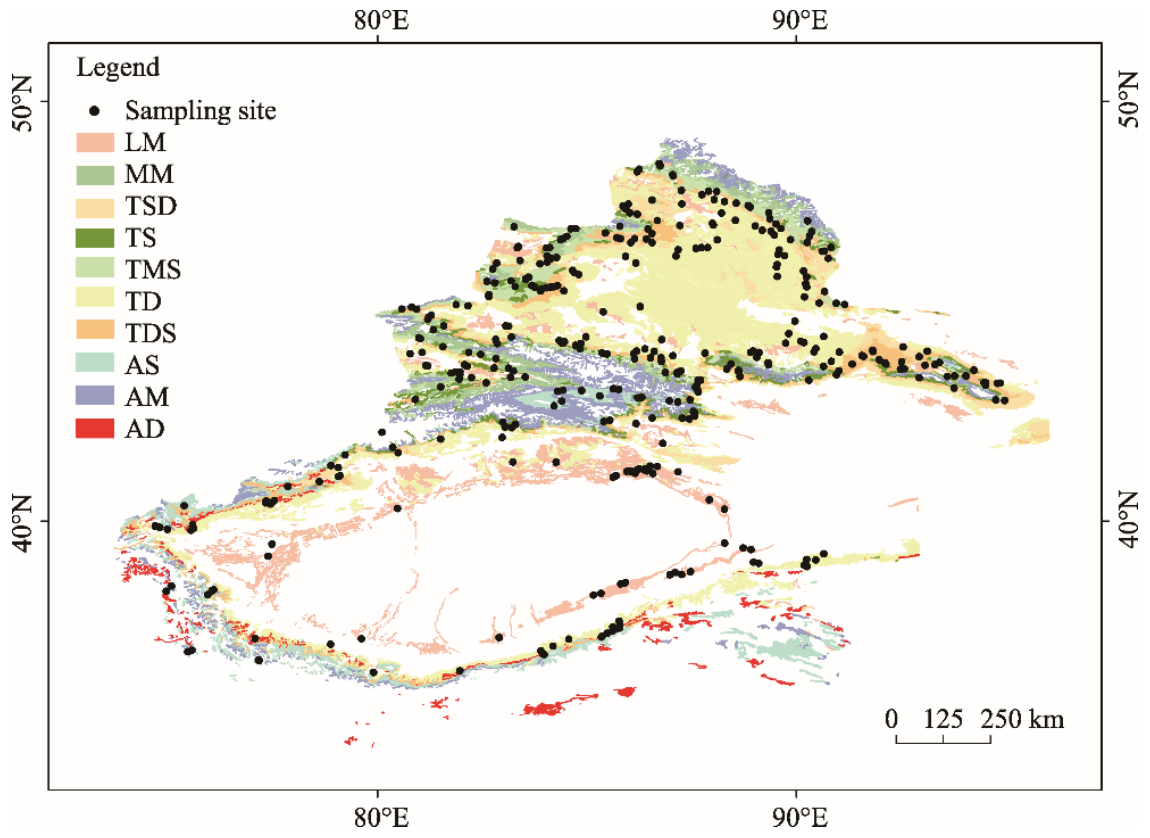

Fig. 1 Spatial distributions of the main grassland types in Xinjiang of Northwest China and locations of the sampling sites in the grasslands. LM, lowland meadow; MM, mountain meadow; TSD, temperate steppe desert; TS, temperate steppe; TMS, temperate meadow steppe; TD, temperate desert; TDS, temperate desert steppe; AS, alpine steppe; AM, alpine meadow; AD, alpine desert. It should be noted that $\mathrm{AD}$ sites were not sampled because of difficulties in reaching them, and AM was removed in this study because of sample size limitation (only 2 sites).

The grasslands in Xinjiang mainly include the following types: lowland meadow (LM), mountain meadow (MM), temperate steppe desert (TSD), temperate steppe (TS), temperate meadow steppe (TMS), temperate desert (TD), temperate desert steppe (TDS), alpine steppe (AS), alpine meadow (AM), and alpine desert (AD) (Fig. 1). In this study, all grassland types were sampled except for AD, which was not sampled because it was distributed at the highest elevation and was difficult to reach (Fig. 1). For the vegetation, the AM was dominated by the genera of Carex and Polygonum, the AS by Stipa and Seriphidium, the LM by Phragmites and Glycyrrhiza, the MM by Ceratocarpus and Carex, the TD by Seriphidium and Ceratocarpus, the TDS by Stipa and Seriphidium, the TMS by Stipa and Carex, the TS by Stipa and Carex, and the TSD by Stipa and Seriphidium (Liu et al., 2016b). The major soil types in the study area were sandy soil, brown desert soil, brown calcic soil, chestnut soil, chernozem, alpine meadow soil and alpine meadow soil.

\subsection{Surveys of SR}

A total of 381 sites were surveyed in the grasslands of Xinjiang, including 2 AM, 18 AS, 36 LM, 6 MM, 127 TD, 84 TDS, 10 TMS, 64 TS and 34 TSD sites. It should be noted that only 2 AM sites were available, which did not meet the requirement for statistical analysis; therefore, AM was not included in the study. The remaining 379 sites covering 8 grassland types were used in further analysis.

SR was surveyed in summers when most grassland species achieve their peak biomass during 2011-2013 (Sala, 2000). The following procedures were used to survey SR at each site: (1) a 100 $\mathrm{m} \times 100 \mathrm{~m}$ sampling area was randomly established, (2) geographic coordinates and elevation of the sampling area were recorded using a portable GPS (eTrex H, Taiwan of China), (3) 3 or 5 quadrats with an area of $1 \mathrm{~m} \times 1 \mathrm{~m}$ for each were randomly set up within the large sample area (Ma et al., 2010), and (4) species names and the density of each species were recorded for each quadrat. The number of species in the quadrats was used to express SR. 


\subsection{Determination of BGB}

After the SR survey, the following procedures were used to measure the BGB for each quadrat at each site. First, 3 (nutrient-poor soil) or 5 (nutrient-rich soil) 7-cm diameter soil cores were randomly taken in the depths of $0-5,5-10,10-20,20-30,30-50,50-70$ and $70-100 \mathrm{~cm}$. For those sites at which soil cores could not be collected because of obstruction by rocks, a soil profile was exposed and the soil cores for different depths were then taken from the profile. For those sites at which the soil thickness was less than $100 \mathrm{~cm}$, the depths were sampled with soil cores until reaching rock. Second, the root system was carefully separated from the soil in the cores. Then, the fresh root was oven dried to a constant weight at $70^{\circ} \mathrm{C}$ for $48 \mathrm{~h}$. Last, the BGB $\left(\mathrm{g} / \mathrm{m}^{2}\right)$ at each site was classified by soil depths: top $(0-20 \mathrm{~cm})$, middle $(20-50 \mathrm{~cm})$ and deep $(50-100 \mathrm{~cm})$ (Mueller et al., 2013).

\subsection{Environmental factors}

In this study, the elevation of each sampling area at each site was recorded using a portable GPS (eTrex H, Taiwan of China). The MAP $(\mathrm{mm}), \operatorname{AMT}\left({ }^{\circ} \mathrm{C}\right)$ and potential evapotranspiration (PET, mm) were used to represent the climatic condition, because variations in SR and BGB are tightly correlated with these climatic factors (Currie and Paquin, 1987; Brown et al., 2004; Cleland et al., 2013). For each site, the MAP and AMT data were extracted from WorldClim (1970-2000; http://www.worldclim.org/) via the "dismo" package in R software (R Development Core Team, 2012; Fick and Hijmans, 2017). The PET data were extracted from the global aridity and PET database (https://cgiarcsi.community/data/global-aridity-and-pet-database) with a resolution of 1 $\mathrm{km} \times 1 \mathrm{~km}$ (Trabucco and Zomer, 2009).

\subsection{Statistical analyses}

To address the first question concerning variations in SR and BGB, we compared grassland types using one-way ANOVA followed by LSD multiple comparisons (Bai et al., 2012). SR and BGB were not transformed in the one-way ANOVA as they were satisfied with requirement of normality (Shapiro-Wilk test) and homogeneity of variance (Levene test). Then, a general linear model was used to explore trends in SR and BGB along environmental gradients, with the linear and quadratic trends fit separately (Su et al., 2017). If both linear and quadratic trends were significant, then the best model was selected using the Akaike information criterion (Burnham and Anderson, 2002). If either a significant linear or a significant quadratic trend was detected, then the significant model was selected directly. The second question concerning the relationship between SR and BGB in different grassland types was also explored using this method and procedure. To address the third question concerning the effects of environmental gradients on the SR-BGB relationship, we used the partial least square path modeling (PLS-PM) to explore direct and indirect effects of elevation, MAP, AMT, PET and SR on BGB. PLS-PM is widely used and particularly suitable for estimating complex cause-effect relationships on strong environmental gradients (Sanchez, 2013; Wang et al., 2016). According to the PLS-PM requirement, we grouped AMT and PET to represent the energy condition, because these two variables are positively correlated (Sanchez, 2013; Wang et al., 2016). The PLS-PM was run with 1000 bootstraps to validate the estimates of path coefficients (which represent the direction and strength of the linear relationships between tested variables) and the accuracy of the PLS-PM was assessed by the goodness of fit statistic (Sanchez, 2013). Finally, the total effect (sum of direct and indirect effects) of each variable was calculated to represent the relative importance to BGB (Sanchez, 2013). These analyses were all conducted in R software, with the PLS-PM conducted using the "plspm" package (R Development Core Team, 2012; Sanchez, 2013).

\section{Results}

\subsection{Variations in SR and BGB among grassland types}

SR varied significantly among grassland types $(P<0.0001$; Fig. $2 \mathrm{a})$. Among the 8 grassland types, SR was higher in TMS (7.67 \pm 1.13$)$ and MM $(7.06 \pm 1.28)$ and lower in TD (1.92 \pm 0.09$)$ and LM $(1.39 \pm 0.14)$ (Fig. 2a). The SR values in TMS and MM were 3-5-fold higher than those in TD and 
LM. Compared with SR, BGB varied differently among grassland types. In the top soil depth (0-20 $\mathrm{cm}$ ), the highest BGB was observed in AS, which had a medium level of SR (3.76 \pm 0.48 ) (Fig. 2b); whereas in the middle $(20-50 \mathrm{~cm})$ and deep $(50-100 \mathrm{~cm})$ soil depths, the highest BGB was observed in LM, which had the lowest SR (1.39 \pm 0.14$)$ (Figs. 2c and d). However, the lowest BGB was always found in TD, irrespective of soil depth (Figs. 2b-d). Because BGB in the $0-20 \mathrm{~cm}$ depth accounted for $48 \%$ of the total BGB in LM and $84 \%$ of the total BGB in MM in the $0-100$ $\mathrm{cm}$ depth, the variations in the total BGB in the $0-100 \mathrm{~cm}$ depth followed that of BGB in the top soil depth $(0-20 \mathrm{~cm})$ in those grasslands (Figs. 2b-e).
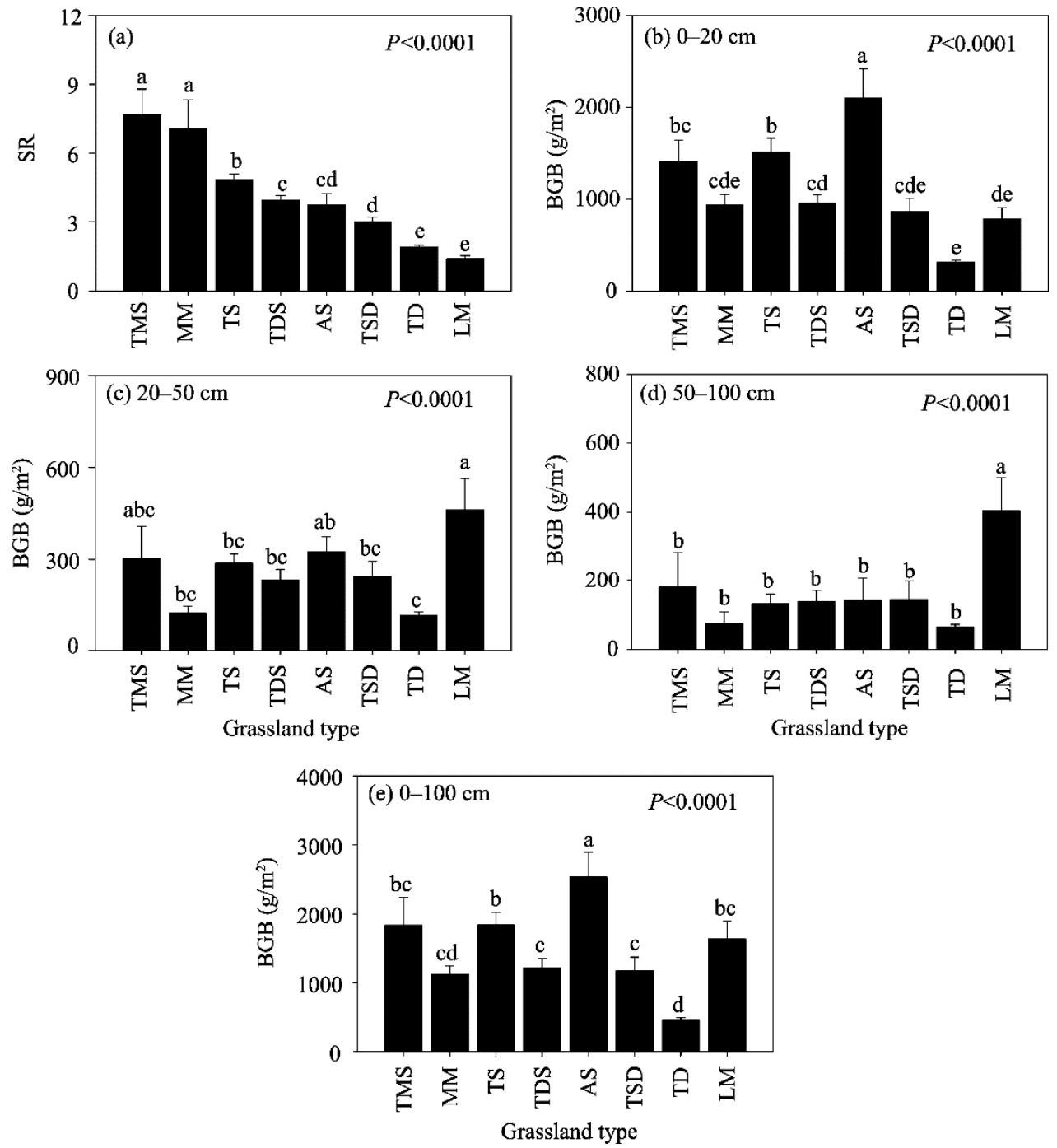

Fig. 2 Variations in species richness (SR; a) and belowground biomass (BGB) in the soil depths of $0-20 \mathrm{~cm}$ (b), 20$50 \mathrm{~cm}(\mathrm{c}), 50-100 \mathrm{~cm}(\mathrm{~d})$ and $0-100 \mathrm{~cm}(\mathrm{e})$ among different grassland types. TMS, temperate meadow steppe ( $n=10)$; MM, mountain meadow $(n=6)$; TS, temperate steppe $(n=64)$; TDS, temperate desert steppe $(n=84)$; AS, alpine steppe $(n=18)$; TSD, temperate steppe desert $(n=34)$; TD, temperate desert $(n=127)$; LM, lowland meadow $(n=36)$. Different lowercase letters above the bars indicate significant differences among grassland types according to LSD test $(P<0.0001)$. The bars mean standard errors.

\subsection{Trends in SR and BGB along environmental gradients}

Changes in SR and BGB in the different soil depths along environmental gradients are shown in Figure 3. Along environmental gradients, SR increased firstly and then decreased with increasing elevation and AMT; further, it was positively associated with MAP and negatively associated with 
PET (Figs. 3a-d). These results indicated that in the grasslands of Xinjiang, the highest SR was in those sites at middle elevations with medium AMT, high MAP and low PET. With increasing elevation, BGB in each soil depth increased positively and linearly, except for BGB in the 50-100 $\mathrm{cm}$ depth (Figs. 3e, i, $\mathrm{m}$ and $\mathrm{q}$ ). The relationship between MAP and BGB shifted from positive in the top soil depth, to negative first and then positive in the middle soil depth, and to negative in the deep soil depth (Figs. 3f, j and n). With increasing AMT and PET, BGB always decreased firstly and then increased, irrespective of soil depth (Figs. $3 \mathrm{~g}, \mathrm{~h}, \mathrm{k}, \mathrm{l}, \mathrm{o}, \mathrm{p}, \mathrm{s}$ and $\mathrm{t}$ ).
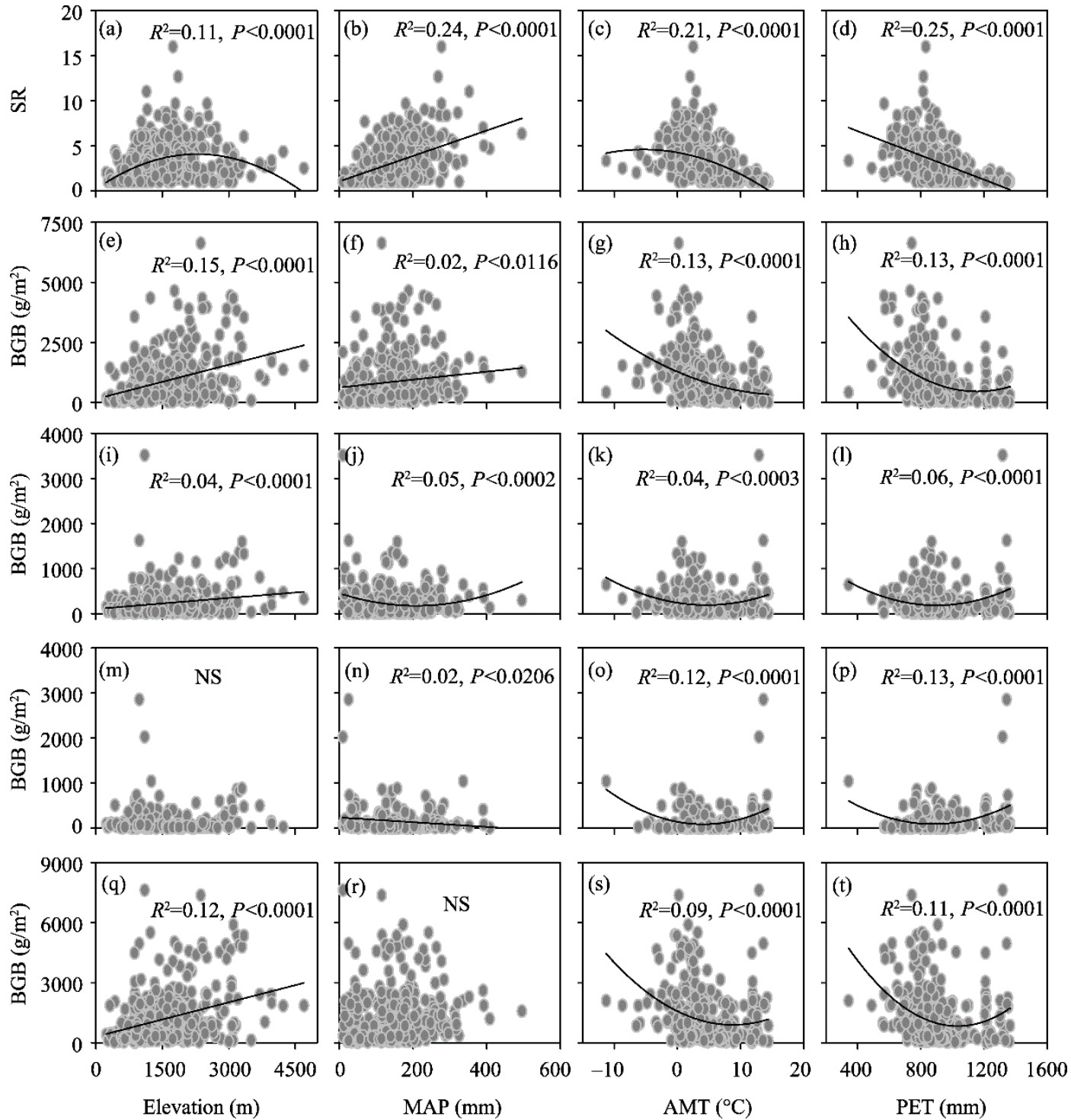

Fig. 3 Changes in SR (a-d; $n=379)$ and BGB in the soil depths of $0-20 \mathrm{~cm}(\mathrm{e}-\mathrm{h} ; n=379), 20-50 \mathrm{~cm}(\mathrm{i}-1 ; n=346), 50-$ $100 \mathrm{~cm}(\mathrm{~m}-\mathrm{p} ; n=211)$ and $0-100 \mathrm{~cm}(\mathrm{q}-\mathrm{t} ; n=379)$ along elevation, mean annual precipitation (MAP), annual mean temperature (AMT) and potential evapotranspiration (PET) gradients in the grasslands of Xinjiang. "NS" indicates no significant relationship.

\subsection{Relationship between SR and BGB}

A unimodal pattern (increased firstly and then decreased) described the relationship between SR and BGB in the top soil depth $(0-20 \mathrm{~cm})$, and a positive correlation described the relationship between SR and BGB in the middle soil depth $(20-50 \mathrm{~cm})$ and total soil depth $(0-100 \mathrm{~cm})$; however, no significant relationship was detected between SR and BGB in the deep soil depth (50$100 \mathrm{~cm}$ ) (Fig. 4). These results suggested that in the grasslands of Xinjiang, the relationship 
between SR and BGB was dependent on soil depth. Furthermore, for the different grassland types, a significant unimodal pattern described the SR-BGB relationship in the top soil depth in TD and TS (Fig. 4a) and in the total soil depth in TS (Fig. 4d).
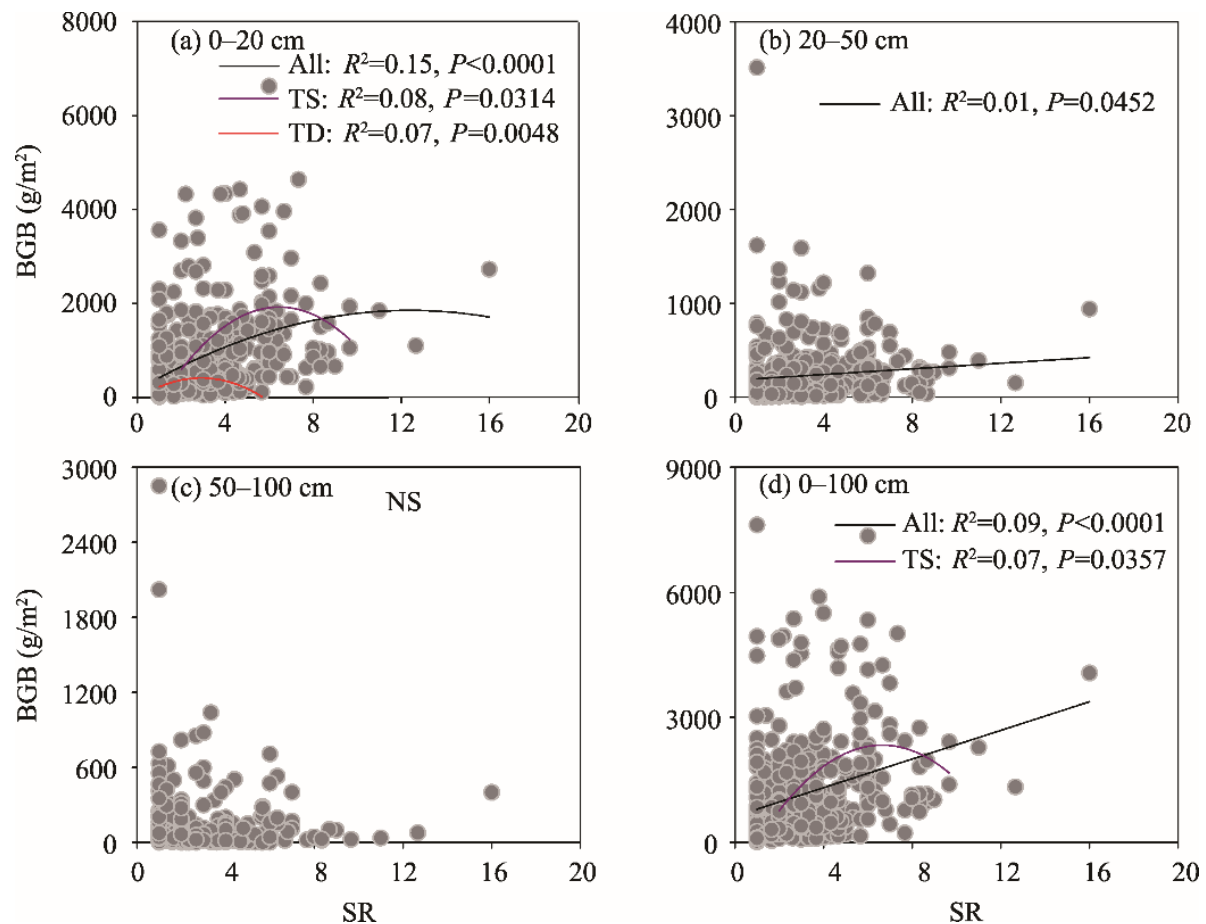

Fig. 4 Relationship between SR and BGB in the soil depths of 0-20 cm (a), 20-50 cm (b), 50-100 cm (c) and 0-100 $\mathrm{cm}$ (d) in the grasslands of Xinjiang. "All" indicates all the grassland types and "NS" indicates no significant relationship. TD, temperate desert; TS, temperate steppe.

\subsection{Effects of environmental factors and SR on BGB}

In the PLS-PM analysis, BGB was significantly affected by a series of direct and indirect effects of environmental factors and SR (Fig. 5). For example, BGB in each soil depth was directly and positively affected by elevation, SR and energy condition (AMT and PET), except for BGB in the top and deep soil depths, for which energy condition and SR, respectively, had no significant influence on BGB (Fig. 5). In addition to the direct effects, BGB was also significantly affected by a series of indirect effects. For example, effects of SR on BGB in the top $(0-20 \mathrm{~cm})$, middle $(20-50$ $\mathrm{cm})$ and total $(0-100 \mathrm{~cm})$ soil depths were indirectly and negatively determined by energy condition, and energy condition in turn was also negatively regulated by elevation and MAP (Fig. 5). Although all these factors had significant direct and indirect effects on BGB, the variations in BGB in the top soil depth and total soil depth were mostly determined by elevation, whereas those in the middle and deep soil depths were mostly affected by energy condition (Fig. 6). SR was the second most influential factor in the regulation of variations in BGB in each soil depth except for the depth of 50-100 cm (Fig. 6).

\section{Discussion}

\subsection{Variations in SR and BGB}

Among the grassland types in Xinjiang, TMS and MM had the highest SR, and TD and LM had the lowest SR, which are consistent with those of previous reports from global grasslands and grasslands on the Tibetan Plateau and in Inner Mongolia, China (Ma et al., 2010; Fraser et al., 2015; Chen et al., 2016). The lowest SR in TD and LM may be partially explained by the harsh 

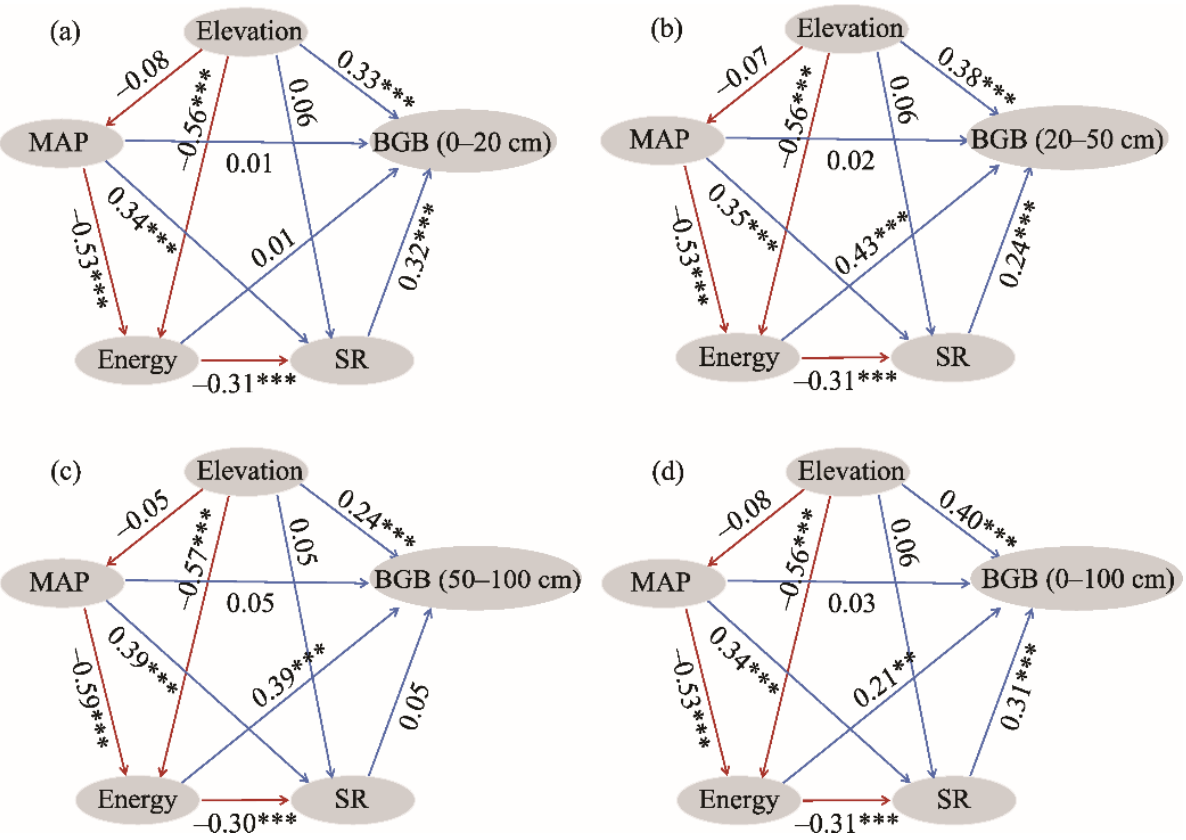

Fig. 5 Direct and indirect effects of energy condition (including AMT and PET), SR, elevation and MAP on BGB in the soil depths of 0-20 cm (a), 20-50 cm (b), 50-100 cm (c) and 0-100 cm (d). *, significance at $P<0.05$ level; **, significance at $P<0.01$ level; ***, significance at $P<0.001$ level. Whole models were assessed using the goodness of fit statistic: 0.52 (a), 0.49 (b), 0.51 (c) and 0.52 (d). Blue and red arrows represented positive and negative effects between connected variables, respectively.
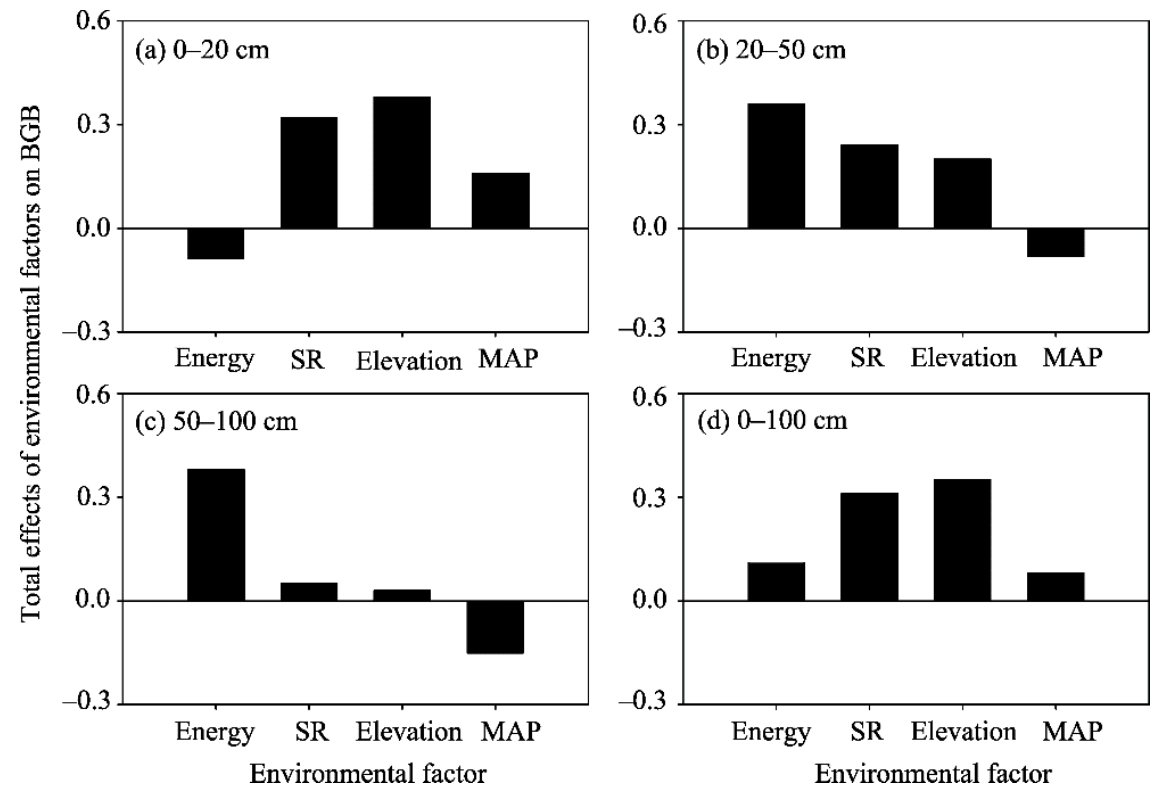

Fig. 6 Total effects of energy condition (including AMT and PET), SR, elevation and MAP on BGB in the soil depths of 0-20 cm (a), 20-50 cm (b), 50-100 cm (c) and 0-100 cm (d)

environmental conditions of relatively low precipitation but high temperature, because only a few species are adapted to survive in those conditions (Keddy, 1992). In TMS and MM, where species growth is primarily limited by low temperature (Callaway, 2007), the high SR was likely the result of facilitation rather than competition dominating the interaction between species (Callaway, 2007).

In the grasslands of Xinjiang, BGB varied differently among grassland types when compared with SR. The highest BGB in the top soil depth in AS is consistent with the result of a previous 
study on Tibetan alpine grassland (Zhu et al., 2017). This result may be due to that species of Stipa, Seriphidium and Carex dominate AS (Liu et al., 2016b) and these species are characterized by a shallow root system with a large proportion of root biomass allocated in the top soil of $0-30 \mathrm{~cm}$ (Chen, 1986). The high BGB in the middle and deep soil depths in LM can be explained by the dominance of Phragmites (Liu et al., 2016b) that has a high-density root system penetrating deeply (Wang et al., 2018).

\subsection{Variations in SR and BGB along environmental gradients}

The change in SR with increasing elevation was described by a unimodal pattern (increased firstly and then decreased), which is consistent with the distribution of species diversity along elevation gradients globally (Guo et al., 2013). The unimodal pattern of SR along elevation gradients can be explained by several mechanisms (Lomolino, 2001). For example, decreases in temperature and soil nutrients and an increase in isolation can reduce SR at high elevations; whereas a low density of native species is associated with low SR at low elevations (Lomolino, 2001). The increase in SR with increasing MAP in the current study is consistent with observations in previous studies in Inner Mongolia of China (Ni, 2003).

BGB also responded differently along different environmental gradients. The increase in BGB with increasing elevation in this study can be explained by slow root turnover at high elevations. For example, the rate of root turnover in AM is relatively low compared with that in tropical grassland (Sun et al., 2018). Along AMT and PET gradients, BGB in the top soil depth decreased firstly and then increased, which is consistent with observations in the grasslands of Tibet, China (Zhu et al., 2017). This phenomenon can be explained by differences in energy condition. Under a low-energy condition (low AMT and PET), the slow root turnover caused by low temperature results in species maintaining a large root biomass (Sun et al., 2018). Under a high-energy condition, in which species growth is primarily limited by water availability, plants allocate more biomass to roots to obtain soil water and in turn generate a relatively large root biomass (Brouwer, 1983).

\subsection{Relationship between SR and BGB}

In the grasslands of Xinjiang, the relationship between SR and BGB changed with soil depth. It was unimodal in the top soil depth and positive and linear in the middle soil depth, but no significant relationship was detected in the deep soil depth. These findings have suggested that the relationship between SR and BGB was depended on soil depth, which could help settle the debate on whether the relationship between SR and BGB is unimodal or positive (Bessler et al., 2009; Mueller et al., 2013; Zhu et al., 2017; Chen et al., 2018). The unimodal pattern between SR and BGB in the top soil depth indicated that grasslands with high and low SR had a relatively low BGB, but grasslands with middle SR had a high BGB. In the middle soil depth, in which soil nutrient availability is low relative to that in the top soil depth, communities with high SR tend to increase rooting depth to obtain soil nutrients, which in turn leads to a positive relationship between SR and BGB (Mueller et al., 2013). In the deep soil depth, root biomass and density are primarily constrained by physical barriers, such as rocks (Jackson et al., 1996), which may partly explain why no significant relationship was observed between SR and BGB in the deep soil depth in the current study.

\subsection{Effects of environmental factors and SR on BGB}

Vertical zonal distribution characterizes the grasslands in Xinjiang, with elevations that range from 220 to $4700 \mathrm{~m}$ a.s.l. (Xu, 1993). Because of the large difference in elevation, the MAP, AMT and soil depth changed with increasing elevation (Koerner, 2007). Although those variables all had significant effects on $\mathrm{BGB}$, however, our findings showed that elevation was the mostly important factor in regulating BGB variation in the top soil depth $(0-20 \mathrm{~cm})$, which highlighted the importance of elevation in affecting BGB in the grasslands of Xinjiang. It is widely acknowledged that plant growth in high elevations was mainly limited by AMT (Koerner, 2007). Compared with the top soil depth at high elevations, soil temperature in the middle and deep soil depths is relatively high in winter but low in the growing season, which benefits root growth because high 
temperature in winter will accelerate nitrogen mineralization and low temperature in the growing season can extend root life span (Burke and Raynal, 1994). This finding was confirmed by our results as BGB in the middle and deep soil depths was mostly constrained by energy condition (AMT and PET). In addition to elevation and energy condition, SR was the next most important factor affecting variations in BGB. Although the effects of SR on BGB determined from controlled experiments are widely reported and discussed (Bessler et al., 2009; Mueller et al., 2013), this study highlights that in the natural grasslands of Xinjiang, the relationship between SR and BGB is primarily modified by elevation and energy condition.

\section{Conclusions}

In the grasslands of Xinjiang, SR and BGB varied differently among different grassland types. AS had a middle level of SR but the highest BGB in the top soil depth $(0-20 \mathrm{~cm})$; whereas LM had the lowest SR but the highest BGB in the middle $(20-50 \mathrm{~cm})$ and deep $(50-100 \mathrm{~cm})$ soil depths. SR and BGB in the different soil depths were tightly associated with environmental gradients, but the particular forms of trends depended on environmental factors and soil depths. A unimodal pattern was observed between SR and BGB in the top soil depth, but SR was positively related with BGB in the middle soil depth and total soil depth $(0-100 \mathrm{~cm})$. Although MAP, energy condition (AMT and PET) and SR had significant effects on BGB, variations in BGB in the top soil depth and total soil depth were mostly determined by elevation, whereas those in the middle and deep soil depths were mostly affected by energy condition. SR was the next most influential factor in regulating variations in BGB in each soil depth. The findings in this study greatly enhance understanding of the SR-BGB relationship on a broad scale with a diversity of habitats and highlight the importance of environmental factors in the regulations of SR and BGB as well as their interaction in the grassland ecosystems.

\section{Acknowledgements}

This research was supported by the National Natural Science Foundation of China (U1603235, 31660127) and the Tianshan Innovation Team Plan of Xinjiang (2017D14009).

\section{References}

Allan E, Weisser W W, Fischer M, et al. 2013. A comparison of the strength of biodiversity effects across multiple functions. Oecologia, 173(1): 223-237.

Ammer C. 2019. Diversity and forest productivity in a changing climate. New Phytologist, 221(1): 50-66.

Bai Y F, Wu J G, Clark C M, et al. 2012. Grazing alters ecosystem functioning and C:N:P stoichiometry of grasslands along a regional precipitation gradient. Journal of Applied Ecology, 49(6): 1204-1215.

Bessler H, Temperton V M, Roscher C, et al. 2009. Aboveground overyielding in grassland mixtures is associated with reduced biomass partitioning to belowground organs. Ecology, 90(6): 1520-1530.

Brouwer R. 1983. Functional equilibrium: sense or nonsense? Netherlands Journal of Agricultural Science, 31: 335-348.

Brown J H, Gillooly J F, Allen A P, et al. 2004. Toward a metabolic theory of ecology. Ecology, 85(7): 1771-1789.

Burke M K, Raynal D J. 1994. Fine-root growth phenology, production, and turnover in a northern hardwood forest ecosystem. Plant and Soil, 162(1): 135-146.

Burnham K P, Anderson D R. 2002. Model Selection and Multimodel Inference: A Practical Information-Theoretic Approach. Berlin: Springer Press, 1-488.

Callaway R M. 2007. Positive Interactions and Interdependence in Plant Communities. Dordrecht: Springer Press, 1-404.

Chen D M, Cheng J H, Chu P F, et al. 2016. Effect of diversity on biomass across grasslands on the Mongolian Plateau: contrasting effects between plants and soil nematodes. Journal of Biogeography, 43(5): 955-966.

Chen S H. 1986. Root System Type of Grassland Plants in Inner Mongolia. Hohhot: People's Publishing of Inner Mongolia, 1-284. (in Chinese)

Chen S P, Wang W T, Xu W T, et al. 2018. Plant diversity enhances productivity and soil carbon storage. Proceedings of the National Academy of Sciences of the United States of America, 115(16): 4027-4032.

Cleland E E, Collins S L, Dickson T L, et al. 2013. Sensitivity of grassland plant community composition to spatial vs. temporal variation in precipitation. Ecology, 94(8): 1687-1696. 
Currie D J, Paquin V. 1987. Large-scale biogeographical patterns of species richness of trees. Nature, 329(6137): 326-327.

De-Boeck H J, Lemmenss C M H M, Zavalloni C, et al. 2008. Biomass production in experimental grasslands of different species richness during three years of climate warming. Biogeosciences, 5(2): 585-594.

Fick S E, Hijmans R J. 2017. Worldclim 2: New 1-km spatial resolution climate surfaces for global land areas. International Journal of Climatology, 37(12): 4302-4315.

Fraser L H, Pither J, Jentsch A, et al. 2015. Worldwide evidence of a unimodal relationship between productivity and plant species richness. Science, 349(6245): 302-305.

Guo Q F, Berry W L. 1998. Species richness and biomass: dissection of the hump-shaped relationships. Ecology, 79(7): $2555-2559$.

Guo Q F, Kelt D A, Sun Z Y, et al. 2013. Global variation in elevational diversity patterns. Scientific Report, 3: 3007, doi: 10.1038/srep03007.

Hooper D U, Chapin F S, Ewel J J, et al. 2005. Effects of biodiversity on ecosystem functioning: a consensus of current knowledge. Ecological Monographs, 75(1): 3-35.

Isbell F, Gonzalez A, Loreau M, et al. 2017. Linking the influence and dependence of people on biodiversity across scales. Nature, 546(7656): 65-72.

Jackson R B, Canadell J, Ehleringer J R, et al. 1996. A global analysis of root distributions for terrestrial biomass. Oecologia, 108(3): 389-411.

Keddy P A. 1992. Assembly and response rules: two goals for predictive community ecology. Journal of Vegetation Science, 3(2): 157-164.

Koerner C. 2007. The use of 'altitude' in ecological research. Trends in Ecology and Evolution, 22(11): 569-574.

Liu L L, Cheng J H, Liu Y H, et al. 2016a. Relationship of productivity to species richness in the Xinjiang temperate grassland. PloS ONE, 11(4): E0154026, doi: 10.1371/journal.pone.0154026.

Liu L L, Sheng J D, Cheng J H, et al. 2016b. Relationship between plant species characteristics and climate factors in different grassland types of Xinjiang. Acta Prataculturae Sinica, 25(7): 1-12. (in Chinese)

Lomolino M V. 2001. Elevation gradients of species-density: historical and prospective views. Global Ecology and Biogeography, 10(1): $3-13$

Loreau M. 2000. Biodiversity and ecosystem functioning: recent theoretical advances. Oikos, 91(1): 3-17.

Ma W H, He J S, Yang Y H, et al. 2010. Environmental factors covary with plant diversity-productivity relationships among Chinese grassland sites. Global Ecology and Biogeography, 19(2): 233-243.

Mueller K, Tilman D, Fornara D A, et al. 2013. Root depth distribution and the diversity-productivity relationship in a long-term grassland experiment. Ecology, 94(4): 787-793.

Ni J. 2003. Plant functional types and climate along a precipitation gradient in temperate grasslands, north-east China and south-east Mongolia. Journal of Arid Environments, 53(4): 501-516.

R Development Core Team. 2012. R: A Language and Environment for Statistical Computing. Vienna: R Foundation for Statistical Computing.

Sala O E. 2000. Global biodiversity scenarios for the year 2100. Science, 287(5459): 1770-1774.

Sanchez G. 2013. PLS Path Modeling with R. Berkeley: Trowchez Editions.

$\mathrm{Su}$ R, Cheng J, Chen D, et al. 2017. Effects of grazing on spatiotemporal variations in community structure and ecosystem function on the grasslands of Inner Mongolia, China. Scientific Reports, 7(1): 40, doi: 10.1038/ s41598-017-00105-y.

Sun Y F, Wan H W, Zhao Y J, et al. 2018. Spatial patterns and drivers of root turnover in grassland ecosystems in China. Chinese Journal of Plant Ecology, 42(3): 337-348. (in Chinese)

Tilman D. 1999. The ecological consequences of changes in biodiversity: a search for general principles. Ecology, 80(5): 14551474.

Trabucco A, Zomer R J. 2009. Global Aridity Index (Global-Aridity) and Global Potential Evapo-Transpiration (Global-PET) Geospatial Database. CGIAR Consortium for Spatial Information. [2019-04-20]. http://www.csi.cgiar.org.

Turnbull L A, Isbell F, Purves D W, et al. 2016. Understanding the value of plant diversity for ecosystem functioning through niche theory. Proceedings of the Royal Society B-Biological Sciences, 283(1844): 1-9.

Xu P. 1993. Grassland Resources and Their Utilization in Xinjiang. Urumqi: Xinjiang Science and Technology Publishing Press, 1400. (in Chinese)

Wang J J, Pan F, Soininen J, et al. 2016. Nutrient enrichment modifies temperature-biodiversity relationships in large-scale field experiments. Nature Communications, 7: 13960, doi: 10.1038/ncomms13960.

Wang J W, Zhao C Z, Zhao L C, et al. 2018. Response of root morphology and biomass of Phragmites australis to soil salinity in inland salt marsh. Acta Ecologica Sinica, 38(13): 4843-4851. (in Chinese)

Zhu G L, Li J, Wei X H, et al. 2017. Longitudinal patterns of productivity and plant diversity in Tibetan alpine grasslands. Journal of Natural Resources, 32(2): 210-222. (in Chinese) 